\title{
Stress in der Tierarztpraxis - (wie) lässt er sich managen?
}

\author{
Lisa Leiner
}
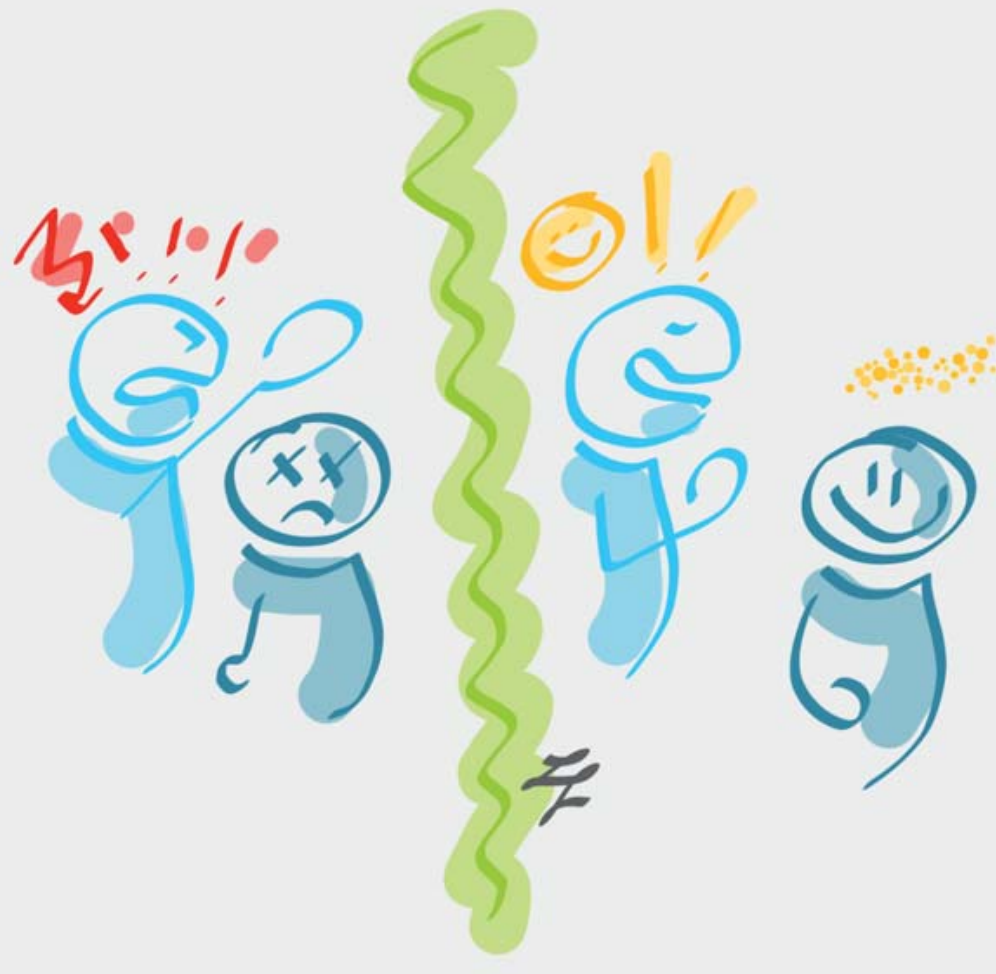

Andauernde Gefühle von Stress, ob selbst verursacht oder von außen „auferlegt“, und das Arbeiten unter Zeit- und Leistungsdruck machen krank. Man hat das Gefühl, man kommt nicht mit der Erledigung der Aufgaben hinterher. Es beginnt mit mehr Kaffee, Energy-Drinks, weniger Schlaf und mündet in Phasen der Verzweiflung oder Blackout. Mit diesen Empfindungen werden nicht nur praktizierende Tierärzte während ihrer beruflichen Laufbahn konfrontiert. Auch Veterinärmedizinstudierende erfahren bereits früh, was es heißt, unter Stress zu arbeiten [8].

\section{Gestiegene Ansprüche}

Der Anspruch an die tierärztliche Leistung und an die Personen, die hinter der Profession des Tierarztes stecken, ist durch vielfältige Veränderungen in der „Tierhalterwelt“ so hoch wie nie zuvor. Neue Unternehmen sprießen sprichwörtlich aus dem Boden, um dem modernen Tierhalter einen noch ausgefalleneren oder individuelleren Service für das eigene Haustier bieten zu können - ob in der Gesundheitsüberwachung, der bedarfsgerechten Ernährung oder dem gemeinsamen Urlaub [3]. Auch die Auseinandersetzung mit Konkurrenzmärkten, die keine tierärztliche Approbation erfordern, oder gängige „Kosten-Diskussionen“ mit Patientenbesitzern [5] nehmen immer größeren Raum ein. Dass all diese Faktoren neben dem ohnehin stressigen Arbeitsalltag eine deutliche Belastung darstellen können, liegt vermutlich auf der Hand.

\section{Stressige Fragen}

Wie kann man Stress „managen“, wenn man keinen Einfluss auf externe Gegebenheiten hat? Zum Beispiel darauf, wie viele Patientenbesitzer die Praxis betreten oder nachts anrufen? Oder man wieder mit Patientenbesitzern über die Tabletteneingabe für den Hund oder die Abrechnung von Leistungen diskutieren muss? Oder sich der Pferdebesitzer beschwert, dass er 30 Minuten im Stall warten musste?

Wie kann man mit Stressgefühlen umgehen, die entstehen, wenn die Wut nach schlechten Bewertungen in Onlineportalen, 
spontaner Kündigung von Mitarbeitern oder sonstigen Ausfällen abgeklungen ist? Wenn man plötzlich vor einem Berg voller Gefühle der Frustration, Angst und Verzweiflung steht?

Um eines vorweg zu nehmen: Es gibt kein „Patentrezept“ gegen Stress. Jeder muss selbst Wege finden, die funktionieren. Und dies erfährt man nur nach dem Trialand-Error-Prinzip. Das eine hat super geholfen, das andere überhaupt nicht. Nur eines sollten Sie nie: stehen bleiben.

\section{Folgen}

Die Reaktionen auf stressige Situationen fallen nicht nur individuell, sondern auch situationsbedingt unterschiedlich aus. Der Eine behauptet, er könne unter „Stress“ effektiver arbeiten, der Nächste sitzt nach einem 12-Stunden-Dienst erschöpft in der Ecke und fragt sich, wie er noch so einen Tag überstehen soll. Auch Dauerstress führt zu unterschiedlichen Reaktionen: Der Eine fällt in eine Lethargie, der Andere in ungerichteten Aktionismus. Der Eine gesteht sich ein, dass es so nicht weitergehen kann, der Andere rennt blind weiter bis zum Umfallen.

Fakt ist: Man könnte viele krankheitsbedingte Ausfälle verhindern, würde man mehr präventive Maßnahmen einführen. Dazu gehört nicht nur das Einhalten von (gesetzlich) geregelten Arbeits- und Ruhezeiten, sondern auch Aspekte wie Wertschätzung, eine gute Kommunikation und das Zugestehen von Pausen (die leider oft noch immer als „Schwäche“ bewertet werden) $[2,4]$.

Wer effektiv, erfolgreich, motiviert und langfristig diesen Beruf ausüben möchte, sollte den Blick einmal von seinen Patientenbesitzern abwenden und auf sich selbst schauen. Denn diese „Ich-bezogene-Aufmerksamkeit" und das Nehmen von Auszeiten sind die Basis für eine gute tierärztliche Arbeit. Wer gestresst und übellaunig ist, kann weder gute Diagnosen stellen, noch eine dauerhafte Kundenzufriedenheit aufbauen.

\section{„Externer" Stress}

Sowohl im beruflichen, als auch im privaten Leben können Dinge passieren, die man nicht beeinflussen kann. Was Sie aber beeinflussen können, ist, wie Sie darauf reagieren. Und darum geht es unter anderem beim Stressmanagement: um die persönliche Einstellung zu Dingen, die man nicht ändern kann.

Eine Akzeptanz dieser unveränderlichen Situationen hat nichts mit Resignation zu tun. Sondern eher mit Resilienz: mit Zuversicht, mit Selbsteinschätzung und Selbstwirksamkeit sowie natürlich Selbstvertrauen. Es geht darum, seine persönliche Energie in Aufgaben und Gedanken zu investieren, die lösungsorientiert sind, anstelle sich seinem Ärger, seinem Frust und damit einer Opferrolle hinzugeben. Denn dies ist pure Zeit- und Ressourcenverschwendung. Und von Zeit haben wir ja bekanntlich nicht sehr viel - im Gegensatz zu den Ressourcen. Aber diese muss man manchmal erst „aufspüren“. Dazu später mehr.

Der Umgang mit „externen Stressfaktoren“ hängt hauptsächlich mit der inneren $\mathrm{Hal}$ tung zusammen. Alles Weitere ist ein Managementproblem, denn natürlich kann ich meine Termine im 10-Minuten-Takt ohne Puffer oder Pause vergeben. Dann muss ich mich allerdings nicht wundern, wenn der Tag am Ende in Stress ausartet.

\section{Resilienz}

Wie kann man Resilienz entwickeln und was bedeutet dies überhaupt? In der Definition nach Brockhaus bezeichnet Resilienz „die psychische Widerstandsfähigkeit von Menschen, die es ermöglicht, selbst widrigste Lebenssituationen und hohe Belastungen ohne nachhaltige psychische Schäden zu bewältigen“ [1]. In der Literatur (oder bei „Dr. Google“) werden Sie einige Versionen und „Bausteine“ der Resilienz finden. Ich beziehe mich hier auf sieben Resilienzfaktoren, angelehnt an Reivich u. Shatté [7]. Diese wären Optimismus, Selbstwirksamkeit, Kausalanalyse, Empathie, Emotionssteuerung, Impulskontrolle und Zielorientierung.
Resilienz ist also der Aufbau einer inneren Stärke und hat viel mit Wertschätzung zu tun, dem verantwortungsvollen Umgang mit sich selbst und anderen sowie dem Vermeiden einer lähmenden „Opferrolle“. Es geht um lösungsorientiertes Arbeiten, Flexibilität und den Mut, vielleicht auch etwas unkonventioneller zu denken, anstelle sich hinter seinen Scheuklappen zu verstecken und nur einen einzigen richtigen Weg zu sehen, der vielleicht nie funktionieren wird.

\section{Optimismus}

Vermutlich ein Begriff für jeden. Hier geht es aber um realistischen Optimismus und nicht um das Tragen einer „rosa Brille“. Leben wir im Dauerstress, so fällt es uns irgendwann sehr schwer, die guten und positiven Seiten in Situationen zu erkennen. Wir nehmen eher Kritik wahr als Lob. In diesen Situationen hilft es explizit sich klar zu machen, welche guten Seiten vielleicht auch negative Erlebnisse haben oder haben könnten. Dies ist die Art des Optimismus, der hier gemeint ist.

\section{Selbstwirksamkeit}

Ja! Ich schaffe das! Ob es der Anfangsassistent ist, der seine erste Hündin alleine kastriert, die ersten schweren Wochen und Monate nach einer Praxiseröffnung oder das Überstehen schwieriger Phasen in der Selbstständigkeit. Mit jeder Situation, welche Sie erfolgreich meistern, trainieren Sie Ihre Selbstwirksamkeit, denn dann wissen Sie: Das habe ich schon einmal geschafft, das schaffe ich wieder!

\section{Kausalanalyse}

Ein gern vernachlässigter Punkt. Auch kein einfacher Faktor, denn er erfordert ein gewisses Maß an Objektivität. Er beinhaltet, dass man sich ganz konkret mit dem „Warum“ beschäftigt: Welche ursächlichen Zusammenhänge haben zu dieser oder jener Situation geführt? Warum ist etwas passiert, das mir oder vielleicht auch anderen geschadet hat? Wie kann ich das nächstes Mal vermeiden? 


\section{Empathie}

Was denken oder fühlen meine Patientenbesitzer? Warum gibt es „Zickereien“ im Team? Weswegen hat mich mein Chef gerade so angebrüllt? Lag es an mir, oder steckt hinter dieser Emotionalität vielleicht mehr? Die Fähigkeit, sich in die Gefühlswelt anderer Menschen hineinzuversetzen, ist ein wesentlicher Aspekt der tierärztlichen Tätigkeit. Dies gilt nicht nur im Rahmen der Kundenbindung, sondern auch für den $\mathrm{Zu}$ sammenhalt im Team.

\section{Emotionssteuerung}

Es klingt etwas abwegig: Die „Steuerung“ von Emotionen. Man könnte vielleicht auch von einem „Umlenken“ sprechen. Dem Umlenken von negativen Gefühlen in Gefühle, die angenehmer, wenn nicht gar positiv sind. Das bedeutet nicht, dass Menschen, die ihre Emotionen gut steuern können, keine negativen Gefühle mehr haben oder sich nicht über andere Menschen ärgern. Aber man lernt, diesen Ärger schneller „abzuschütteln“. Es geht eher darum, sich selbst zu sagen: „Ich möchte glücklich sein. Ich möchte mich wohlfühlen.“

\section{Impulskontrolle}

Dieser Begriff ist vielleicht leichter zu verstehen, wenn man den Begriff der „Impulskontrollstörung“ beschreibt: Nägel kauen, Frust-Shopping, Spielsucht bis hin zur Selbstverletzung oder Bulimia nervosa. Es geht um Ungeduld, um ein Handeln ohne Überlegung. Im Umkehrschluss geht es bei der Impulskontrolle darum, Affekte zu kontrollieren oder umzulenken. Statt einem Wutanfall wird eine Atemübung trainiert überspitzt gesagt.

\section{Zielorientierung}

Wer nicht weiß, wofür er das tut, was er tut, kann auch nicht auf das Getane mit Stolz zurückblicken und sagen: Ich habe es geschafft! Ist es das „Große Ganze“, was Sie erreichen wollen? Wie hoch sind Ihre Erwartungen an sich selbst? Mit welchem Gefühl möchten Sie abends ins Bett gehen? Dass
Sie wieder einen „kleinen Schritt“ in die richtige Richtung gelaufen sind? Auch kleine Schritte können sehr befriedigend sein wenn man sie wahrnimmt und wertschätzt.

\section{Aufbauarbeit}

Seine persönliche „innere Stärke“ zu finden ist nicht unbedingt einfach. Bei manchen wird tatsächlich ein „Schalter umgelegt“ und plötzlich ist alles „klar“. Andere kämpfen lange Jahre damit, eine Resilienz aufzubauen und müssen mit vielen Rückschlägen zurechtkommen.

Eine „Schritt-für-Schritt-Anleitung“ für Anfänger gibt es leider nicht. Also, wo fängt man an? Jeder empfindet Stress anders, jeder reagiert anders, jeder ist anders. Was kann man also raten, wenn es so viele individuelle Unterschiede gibt? Im Grunde nur eines: Packen Sie das Problem an der Basis. Und das bedeutet: manchmal intensives Schaufeln und Graben, manchmal mit Splittern in den Händen, aber am Ende ist dieser Aufwand eine langfristige und positive Investition in die eigene Zukunft und Gesundheit. Und hier kommen Ressourcen ins Spiel.

\section{Ressourcen}

Ressourcen sind Hilfsmittel, die dazu dienen, unangenehme Emotionen wieder in angenehme $\mathrm{zu}$ wandeln. Hierbei unterscheidet man innere Ressourcen, wie Erinnerungen an schöne oder erfolgreiche Momente im Leben, die ohne äußere Einflüsse zu positiven Gefühlen führen. Sowie äußere Ressourcen, wie Familienmitglieder, Freunde oder andere soziale Kontakte sowie Orte, an denen man sich wohl fühlt. Jeder kann dabei auf eigene Ressourcen zurückgreifen, die individuell, vielfältig und in unterschiedlichen Situationen zum Einsatz kommen. Ob es sich um persönliche Krisen handelt, um Auseinandersetzungen mit Mitmenschen oder Belastungen bei der Arbeit. Auch körperliche Fitness und das Aufrechterhalten von geistigen Fähigkeiten stellen Ressourcen dar.

Vielleicht fallen Ihnen beim Lesen dieser Zeilen erste Ressourcen ein, unterstützend hilft auch immer eine „Positivliste“, mit
Dingen, die positive Gefühle bei Ihnen auslösen. Wenn der Alltag dann mal wieder zu schwer war und Ihr Kopf mit Denken aufgehört hat, können Sie sich diese Liste vornehmen und einfach mal durchgehen, was Ihnen dann akut helfen könnte.

\section{Rituale}

Auch Rituale können eine stetige Ressource darstellen. Verbinden Sie eine Gewohnheit mit einer gewählten Aktion - und konditionieren Sie so Ihren Kopf und Körper Schritt für Schritt um. Und wenn es nur ein erstes kleines Ritual ist, z.B.: Jeden Morgen, wenn ich meine heiße Kaffeetasse in der Hand halte, schließe ich die Augen, fühle die Wärme in den Händen und sage mir: „Das wird heute ein guter Tag." Dann lächele ich und atme fünfmal tief durch. Sie werden sehen, am Ende ist es die Summe der kleinen Glücksmomente, die Ihnen helfen werden, über den Tag zu kommen [4].

\section{Prävention}

Neu gewonnene Rituale und Ressourcen helfen womöglich über berufliche und persönliche Unzufriedenheit sowie manche externe Stressoren hinweg. Trotz aller „guten Vorsätze“ kann jedoch eine „gescheiterte“ Kommunikation mit Mitmenschen zu einer erhöhten Cortisol-Ausschüttung mit nachfolgender Explosionsgefahr führen. $\mathrm{Ob}$ es sich dabei um Patientenbesitzer oder Mitarbeiter handelt, spielt kaum eine Rolle. In manchen Situationen wird man schlicht „auf dem falschen Fuß“ erwischt.

Die Kommunikation per se beinhaltet bereits ein hohes Maß an Interpretationsmöglichkeit. Eine falsche Interpretation von Aussagen führt zu Missverständnissen und damit verbunden durchaus auch zu falschen Reaktionen oder Handlungen. Diese Irrtümer können, in einer Kette von weiteren Missverständnissen, bis hin zum Mobbing am Arbeitsplatz führen. Die Folgen davon sind neben psychischen Auswirkungen meist auch ein Ausscheiden des Betroffenen aus dem Arbeitsverhältnis [6].

Kommunikations- und Konfliktfähigkeit sind Kompetenzen, die nicht nur dann trai- 


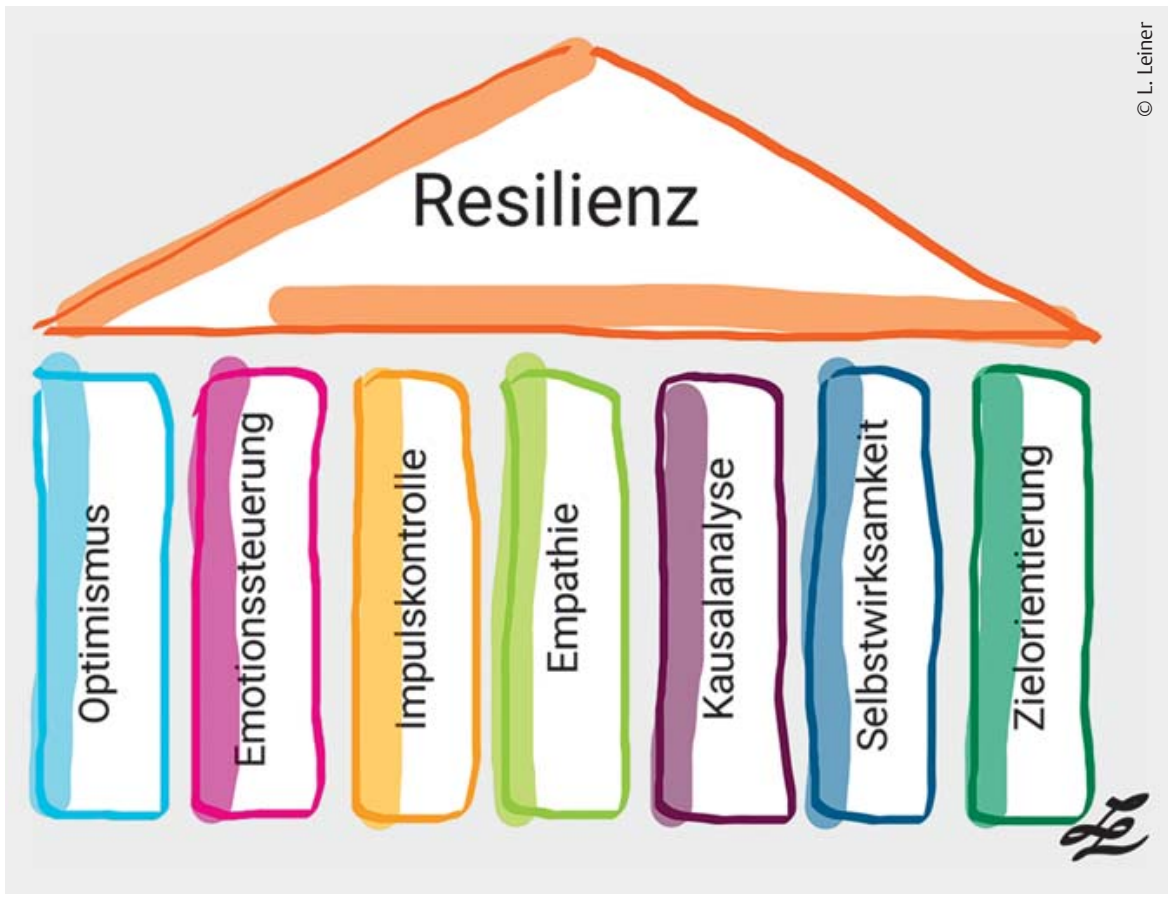

Abb. 1 Eine gute und offene Kommunikationskultur ist die beste Stressprävention für das ganze Team!

niert werden sollten, wenn man bereits Probleme mit anderen Menschen hat. Es sollte als eine grundsätzliche Kompetenz angesehen werden, die es zu fördern gilt.

\section{Gute Kommunikation}

Was ist das Geheimnis einer guten Kommunikation? Eigentlich recht einfach: Lassen Sie keine Interpretationsmöglichkeiten zu und bleiben Sie auf Augenhöhe. Jedoch ist dies leichter gesagt als getan. Nicht von ungefähr bevölkern Kommunikationsseminare die Listen der Fortbildungsanbieter. Nicht umsonst werden Coaches klinik- und praxisintern gebucht, um die Kommunikationsfähigkeit von Mitarbeitern (und Führungskräften) zu schulen.

Interessanterweise spielt dabei die Ebene Tierarzt-Patientenbesitzer eine eher untergeordnete Rolle. Zwar muss man auch hier lernen, sich nicht von „Dr. Google“ das Zepter aus der Hand nehmen zu lassen und seine Leistung entsprechend darzustellen, aber um vor allem in größeren Teams diese
Ebene der Kommunikation erfolgreich (und umsatzstark) umzusetzen, bedarf es erst einer „internen Arbeit“.

\section{Gute Führung}

Das Team hinter dem Tierarzt und vor allem die Führungsebene nehmen ständig Einfluss darauf, wie in der Sprechstunde am Ende kommuniziert wird. Gibt es Querelen und Streitereien im Team, gibt es immer Möglichkeiten, sich aus dem Weg zu gehen (notfalls den Dienstplan dementsprechend ändern). Aber die Führungsebene, die ist omnipräsent.

Ob es um Dienstpläne geht oder um das korrekte Abrechnen von Leistungen. In den allermeisten Fällen würde man sehr viel Stresspotenzial aus dem tierärztlichen Alltag abziehen können, wenn die Führungsebene eine gute Kommunikation mit der Mitarbeiterebene pflegen würde. Was aber bremst? Harmoniebedürftigkeit, Zeitmangel, Angst vor Entlassung, unangenehme Konfliktgespräche oder gar Mobbing. Wer es allerdings schafft, über Hierarchieebenen hinweg eine gute Kommunikationskultur zu etablieren, kann neben dem Aufbau einer persönlichen Resilienz auf einer weiteren Ebene aktiv etwas gegen ein drohendes Stressempfinden tun.

\section{Literatur}

1 Brockhaus Online. Im Internet: https://demo. brockhaus.de/enzyklopaedie/resilienzpsychologie

2 Deutsche Gesetzliche Unfallversicherung (DGUV). DGUV Report 1/2012. Schichtarbeit - Rechtslage, gesundheitliche Risiken und Präventionsmöglichkeiten. Im Internet: http://publikationen.dguv.de/dguv/pdf/ 10002/iag-schicht-1.2012.pdf

3 Kramer. START-UP-BOOM ERREICHT DIE TIERWELT. vetjournal Juli/August 2017-70. JG Im Internet: http://www.tieraerzteverlag. at/fileadmin/dokumente/VET_AUSGABE_0708-2017_ONLINE.pdf

4 Leiner L. Stress- und Zeitmanagement für Tierärzte. Schattauer GmbH 2017

5 Meyer. Die Stellung des Tierarztes in unserer Gesellschaft - vom „Abzocker“ zum „Allwissenden"? Im Internet: https://www.vetstage. de/blog/die-stellung-des-tierarztes-inunserer-gesellschaft-vom-abzocker-zumallwissenden/

6 Olson. Mobbing am Arbeitsplatz - wie es dazu kommt. Im Internet: https://www. vetstage.de/blog/mobbing-am-arbeitsplatzwie-es-dazu-kommt

7 Reivich $u$. Shatté. The Resilience Factor. New York, Broadway Books 2002

8 Rogoll. Tiermedizin: Das stressigste Studium in Deutschland - Teil 1. Im Internet: https:// www.vetstage.de/blog/tiermedizin-dasstressigste-studium-in-deutschland-teil-1/

\section{Online}

https://doi.org/10.1055/s-0043-119499

\section{Verfasserin}

Dr. Lisa Leiner

VetStage $\mathrm{GmbH}$

Potsdamer Platz 1

10785 Berlin

lleiner@vetstage.de

www.vetstage.de 